Rapport - Société canadienne d'histoire de l'Église catholique

\title{
Le cardinal Ignace Persico, capucin, curé de Sillery et sa mission secrète au Canada
}

\section{Gabriel-Marie Dumas}

Volume 32, 1965

URI : https://id.erudit.org/iderudit/1007326ar

DOI : https://doi.org/10.7202/1007326ar

Aller au sommaire du numéro

\section{Éditeur(s)}

Les Éditions Historia Ecclesiæ Catholicæ Canadensis Inc.

\section{ISSN}

0318-6148 (imprimé)

1927-7075 (numérique)

Découvrir la revue

\section{Citer cet article}

Dumas, G.-M. (1965). Le cardinal Ignace Persico, capucin, curé de Sillery et sa mission secrète au Canada. Rapport - Société canadienne d'histoire de l'Église catholique, 32, 11-19. https://doi.org/10.7202/1007326ar

Tous droits réservés (C) Les Éditions Historia Ecclesiæ Catholicæ Canadensis Inc., 1966
Ce document est protégé par la loi sur le droit d'auteur. L'utilisation des services d'Érudit (y compris la reproduction) est assujettie à sa politique d'utilisation que vous pouvez consulter en ligne.

https://apropos.erudit.org/fr/usagers/politique-dutilisation/ 


\section{Le cardinal Ignace Persico, capucin curé de Sillery et sa mission secrète au Canada}

Le 3 décembre 1873, les paroissiens de Sillery sont témoins d'un événement pour le moins insolite: un évêque capucin d'Italie, Mgr Ignace Persico, déjà rompu aux subtilités de la diplomatie romaine, abandonne son siège épiscopal pour devenir leur curé. $\mathrm{La}$ nouvelle, il va sans dire, fait sensation. Bon nombre de fidèles se montrent honorés et flattés de cette nomination; quelques-uns admirent son humilité et son désintéressement; certains, sa bonhomie et sa distinction: mais d'autres, plus malins et peut-être plus perspicaces, voient en lui un envoyé du SaintSiège chargé d'enquêter sur nos affaires religieuses. Et certes la situation troublée de l'Eglise au Québec permet une telle hypothèse. Ne l'oublions pas : c'est l'époque des difficultés célèbres, pénibles et parfois scandaleuses entre l'Université Laval et sa succursale de Montréal; c'est l'époque des démêlés politico-religieux de 1870 à 1876 relatifs au libéralisme dans la province de Québec; c'est l'époque des frictions entre Mgr Laflèche et Mgr Taschereau sur l'opportunité de diviser le diocèse de Trois-Rivières pour créer celui de Nicolet; c'est l'époque des luttes électorales, avec rebondissement dans la sacristie, entre les conservateurs et les libéraux du Québec; et j'en passe!

Or à ce même moment, dans un climat spirituel, intellectuel et politique en effervescence, ce prélat romain accepte de devenir le pasteur d'une petite paroisse en banlieue québécoise. Même si le but avoué de sa venue au pays est de refaire sa santé délabrée, ne peut-on pas se demander si Rome ne profite pas du séjour de Mgr Persico à Québec pour lui confier le soin de surveiller les agissements parfois déroutants de quelques catholiques notoires, qu'ils soient clercs ou laïcs ?

Mais avant d'aborder cette question, il me tarde de vous présenter brièvement le personnage central de l'affaire et de vous dire quelques mots sur la situation religieuse dans laquelle il est plongé à son arrivée au pays.

Pierre Persico est né à Naples, le 30 janvier 1823. Son père était intendant de l'armée napolitaine et sa mère, de famille noble et en vue, était alliée à la branche italienne des Acton d'Angleterre. En 1839, il prend l'habit des capucins sous le nom d'Ignace ${ }^{1}$. Ordonné prêtre à l'âge de vingt-deux ans, le 22 janvier 1846, dans sa ville natale, il est

1 Analecta Ord. Fr. Min. Cap., XII (1896), Rome, p. 30. 
nommé missionnaire aux Indes où les Capucins dirigent cinq territoires de missions.

Très instruit, zélé, éloquent, riche d'initiative et de bonté, familier avec l'italien, l'anglais, le français, le portugais et l'hindoustani, il a tôt fait sa marque comme orateur, éducateur, directeur de deux journaux anglais dont le Bombay Catholic Examiner qui existe encore ${ }^{2}$. Son évêque, le vénérable Anastase Hartman, capucin suisse, l'obtient pour coadjuteur. Il est sacré par lui à Bombay. le 4 juin 1854; il n'a alors que trente et un ans ${ }^{3}$. Il devient enanite viraire annatolingue d'Agra. dans le nord de l'Inde; et ce sont pour lui huit années débordantes d'activité, de sacrifice et de combats même pour la liberté de l'Eglise catholique en proie au schisme goanais et pour le salut de son troupeau menacé, dans la terrible révolte des Cipayes ${ }^{4}$. Délégué officiel de la hiérarchie indienne à Rome, puis de la Propagande à Londres, il s'y révèle diplomate né, sauvegardant en d'extrêmes difficultés les droits et les biens des catholiques; on lui doit le statut avantageux dont jouit encore l'Eglise aux Indes depuis $1858^{5}$.

En 1861, sa santé chancelante le force à entrer définitivement en Italie où il devient attaché à la Propagande pour les affaires de langue anglaise. En 1867, il se rend aux Etats-Unis et devient, trois ans plus tard, évêque de Savannah en Géorgie ${ }^{6}$. Ici, le temps me manque pour vous décrire son apostolat fructueux dans cette région cruellement éprouvée par la guerre de Sécession. De nouveau, son dur labeur compromet sa santé et il doit abandonner à regret son poste.

A Charleston et à Augusta, Mgr Persico fait la connaissance de l'abbé Louis-Honoré Paquet, professeur de dogme à l'Université Laval, qu'une santé délicate amenait, l'hiver, dans le Sud `. Ils ne tardent pas à se lier d'une amitié qui va durer jusqu'à la mort. En 1869, l'abbé Paquet entraîne pour un mois à Québec son ami qui doit se rendre au premier Concile du Vatican ${ }^{8}$. Le prélat a l'occasion d'admirer alors l'esprit chrétien et le bonheur familial et social de notre peuple. Cette première impression est telle que, deux ans plus tard, il choisit le Canada pour venir y refaire sa santé. Il arrive à Québec le 29 juin 1873 et. pendant cinq mois, il est l'hôte de l'archevêque au palais épiscopal ${ }^{9}$; il rend d'innombrables services à l'Hôtel-Dieu, dans les communautés de la

2 Bishop Hartman, by Rev. Fr. Fulgentius, o.f.m.cap., Allahabad, The Indian Press Ltd., 1946, p. 91.

3 Ibid., p. 227 ss.

4 Ibid., chapitre V. Voir aussi : Relation sur l'Insurrection de l'Inde anglaise, par Mgr Ignace Persico, 8 novembre 1857, 9 p., Arch. Prov., Montréal (dossier Mgr Persico).

5 Fr. Fulgentius, op. cit., p. 145 ss.

6 Semaine religieuse de Québec. Voir aussi : Echos et glanures, par l'abbé Louis-Honoré PAQUeT, Québec, Impr. des Franciscaines de Marie, 1916, p. 329.

7 Ibid.

8 Ibid.

9 Histoire du palais épiscopal de Québec. par Mgr Henri TÊtu, Québec, 1896, p. 214-216. 
ville ${ }^{10}$, et dans les paroisses où il administre les sacrements d'ordre et de confirmation ${ }^{11}$. Le jour de la Toussaint, il est nommé Vicaire Général ${ }^{12}$ puis, le 3 décembre suivant, curé de la paroisse Saint-Colomban de Sillery ${ }^{13}$. Dans l'idée de l'archevêque, cette cure confiée à l'évêque capucin n'est qu'un titre et un moyen pour lui fournir des honoraires convenables à son rang; c'est pourquoi, on lui laisse le choix d'un vicaire qui serait, au besoin, curé d'office ${ }^{14}$.

Mgr Persico, cependant, ne veut pas être un curé honoraire; avec le titre, il accepte aussi la charge, se dépensant sans compter auprès de ses paroissiens. Ainsi, il préside dix-sept réunions régulières de la Fabrique et rédige de sa main les procès verbaux. Il voit à la construction d'une école, à la décoration de l'intérieur de l'église; et pour payer les dépenses, il organise une tombola qui remporte un franc succès avec $2,800.00$ dollars de profit. Il fait 73 baptêmes, 46 mariages et 31 sépultures; il confirme 348 enfants ${ }^{15}$; il transcrit dans les régistres les actes de ses fonctions, allant même jusqu'à dresser la table méthodique de ses régistres. A chaque année, c'est lui qui rédige, de sa main ferme et lisible, les Rapports annuels sur l'état de la paroisse en réponse au questionnaire transmis par l'Ordinaire du lieu ${ }^{16}$.

Deux fois l'an, en compagnie de son vicaire, il visite les écoles et procède annuellement à la visite paroissiale dans les foyers. Il réorganise la bibliothèque et s'évertue à répandre les bonnes lectures chez les jeunes. Le Cahier des prônes dominicaux de la paroisse dont près de la moitié sur 180 sont écrits de sa main nous renseigne sur son zèle pastoral. Parmi tous les faits qu'on peut relever à sa louange, je ne veux en souligner qu'un seul qui est bien de circonstance, puisque nous sommes à Chicoutimi : à quatre reprises au moins, Mgr Persico recommande avec instance à ses fidèles de se montrer généreux envers l'œuvre naissante du Séminaire de Chicoutimi que vient de fonder Mgr Racine ${ }^{17}$. C'est qu'il a à coeur les vocations sacerdotales et s'emploie à les faire éclore partout.

Le 14 octobre 1876, après trois ans passés à Sillery, Mgr Persico doit abandonner sa cure et quitter définitivement le Canada, car Rome

10 Lettre de Mgr Taschereau à la Mère Supérieure des Sœeurs de la Charité de Québec, Québec, 5 novembre 1873. Copie conservée aux archives des pères Capucins à Montréal (dossier Mgr Persico).

11 Histoire de la paroisse Saint-Augustin de Portneuf, par A. BÉchard, Québec, Brousseau, 1885, p. 305 ss. Mgr Persico fit plusieurs autres ordinations, cf. Registre des Ordinations, archevêché de Québec.

12 Archives de l'archevêché de Québec, Registre des nominations, $1^{\text {or }}$ novembre $1873,128^{\mathrm{e}}$ feuillet.

13 Lettre de Mgr Taschereau à Mgr Persico, 3 décembre 1873. Archives de l'archevêché de Québec, Registre des lettres, $\mathrm{n}^{\circ} 30,1871-1874$, p. 652.

14 Mgr Ignace Persico, o.m.cap. (1873-1876), par le père Justin, o.m.cap., dans Almanach de l'Action Sociale Catholique, 1936, p. 38-41.

15 Registre des actes de baptêmes, mariages et sépultures de la paroisse Saint-Colomban de Sillery, tome II, 1866-1880.

16 Rapport du 25 août 1874, du 12 août 1875, du 2 août 1876. Archives de la paroisse de Saint-Colomban, cartable I, p. 163-165.

17 Père Justin, op. cit., p. 39. 
le réclame pour lui confier une importante mission diplomatique aux Indes. Puis il est nommé visiteur apostolique en Irlande, en Ecosse et à Naples. En 1883, il devient' archevêque de Damiette et secrétaire de la Congrégation de la Propagande. Créé Cardinal par Léon XIII le 16 janvier 1893, il décède à Rome le 7 décembre $1895{ }^{18}$.

Sa carrière mouvementée est digne de tout éloge : tour à tour moine capucin, missionnaire et Vicaire Apostolique aux Indes, évêque résidentiel aux Etats-Unis, curé de Sillery, évêque de trois diocèses en Italie, plusieurs fois délégué de la Propagande, archevêque, secrétaire de la Propagande puis cardinal. C'est toute une odyssée que j'ai à peine esquissée devant vous.

De toute cette vie entièrement dévouée au service de l'Eglise, un problème se pose relativement à son séjour au Canada. Nous avons vu que ce prélat fut attiré au pays pour y refaire une santé que des climats tropicaux et des travaux apostoliques épuisants ont durement éprouvée. Est-ce là, cependant, la vraie raison ? En venant à Québec, Mgr Persico ne remplit-il pas une mission diplomatique au nom du Saint-Siège ? Mais, au fait, notre pays a-t-il besoin d'un tel délégué ? Le climat religieux de notre fervente province est-il devenu à ce point troublé que Rome décide d'y envoyer un observateur? Pour le savoir, soulevons furtivement le voile du passé et essayons d'y découvrir quelques indices révélateurs d'une telle situation.

En 1872, le pape Pie IX vient de condamner la théorie du libéralisme catholique. Or à cette époque un parti politique canadien, le parti libéral, s'attire les foudres de l'opposition. Les conservateurs lui attribuent les mêmes idées avancées, donc condamnables, et publient contre lui un manifeste appelé le Programme catholique. Mgr Bourget et Mgr Laflèche approuvent le programme sans restriction, tandis que Mgr Taschereau le rejette; le clergé se rallie, avec plus ou moins d'éclats, à l'une ou l'autre thèse ${ }^{19}$.

Les prêtres du Séminaire de Nicolet, par ailleurs, font bloc contre leur évêque, Mgr Laflèche, pour un motif additionnel. L'évêque de Trois-Rivières veut unir le Séminaire de Nicolet à celui de sa ville épiscopale. Le différend est porté en cour de Rome où il est question de diviser le diocèse pour créer celui de Nicolet. Mgr Laflèche, qui n'est pas homme à fléchir sur les grands conflits nationaux, veut encore moins céder sur son propre terrain. Mais les prêtres de Nicolet, opposés à leur chef sur ce point, embrassent la cause de ses ennemis. Et cette dispute de collège ne tarde pas à se greffer sur une querelle beaucoup plus grande et plus spectaculaire, celle de l'Univrsité ${ }^{20}$.

La querelle entre l'Université Laval et sa succursale de Montréal fait couler beaucoup d'encre et pas toujours pour la cause très honora-

18 Analecta O.F.M.Cap., XII (1896), Rome, p. 31.

19 Mgr Laflèche, par Robert RumiLly, Montréal, édition du Zodiaque, 1938 (coll. Zodiaque, $2^{\mathrm{e}}$ ), $424 \mathrm{p}$., passim. Voir aussi : Le libéralisme (leçons données à

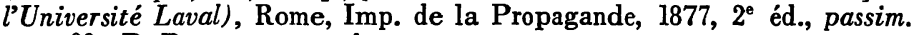

20 R. RUMILLY, op. cit. 
ble de l'obtention d'un doctorat. Mgr Bourget veut avoir sa propre université afin de soustraire les étudiants et les professeurs de la Métropole à l'influence soi-disante libérale de Québec. Les Jésuites prennent parti pour Mgr Bourget et présentent à l'Assemblée Législative un bill les autorisant à fonder une université catholique et française à Montréal. Mais ce projet échoue sur l'intervention de Mgr Taschereau et de ses amis du Parlement ${ }^{21}$.

Comme on le voit, cette dispute connaît les métamorphoses devenues classiques dans la belle Province : elle est d'abord spirituelle, devient par la suite intellectuelle et se transforme en querelle politique. A ce moment, le parti libéral prend de plus en plus d'importance jusqu'à détenir le pouvoir sur la scène provinciale et fédérale. C'en est trop pour des traditionalistes comme Mgr Bourget et Mgr Laflèche. Ces deux évêques, qui se font les défenseurs de l'orthodoxie, soutiennent qu'il revient au clergé d'intervenir dans les élections et d'instruire le peuple sur le choix de leurs chefs et de leurs députés. La chose est d'importance ${ }^{22}$ !

Tous ces problèmes, rendus plus ou moins à leur paroxysme, divisent le peuple et le clergé et provoquent de volumineux et savants rapports que des émissaires, clercs ou laïcs, vont défendre à Rome. Devant ces dossiers souvent contradictoires, la Curie Romaine prit-elle l'initiative d'envoyer un agent secret au Canada ? En tout cas, l'arrivée de Mgr Persico en ces temps troublés nous autorise à le croire, d'autant plus que ce prélat a une réputation de diplomate, et depuis une dizaine d'années le Saint-Siège l'utilise pour ce genre de travail. Et les gens de l'époque, qui ne sont pas dupes, s'accordent à l'affirmer. Il suffit de consulter les journaux contemporains et d'entendre les confidences de quelques intimes du prélat pour nous en convaincre.

L'abbé J. Bigaouette de Québec, qui l'a connu très bien, affirme : * On dit qu'il était délégué secret durant son séjour à Québec pour les affaires de l'Université ${ }^{23}$ ».

L'abbé J.-E. Lizotte, curé de Roberval, qui fut ordonné prêtre par Mgr Persico déclare : « Dans le temps, le bruit courait qu'il avait été envoyé par Rome pour faire une enquête dans certaines affaires religieuses embrouillées ${ }^{24}$.

L'abbé J.-B.-Z. Bolduc écrivait le 20 février 1876 : « J'ai été faire la veillée cette semaine chez Mgr Persico... Je suis persuadé à croire

21 Ibid. Voir aussi : L'Eglise catholique au Canada, précis historique et statistique, $6^{\mathrm{e}}$ éd., par le père Dominique DE SAINT-Denis, capucin, Montréal, éd. Thau, 1956, 269 p. Voir p. 125.

22 Ibid.

23 Lettre de l'abbé J. Bigaouette au père Justin, Québec, 22 février 1934. Arch. Prov. des Capucins (dossier Mgr Persico).

24 Lettre de l'abbé J.E. Lizotte au père Justin, Roberval, 3 mars 1934. Arch. Prov. des Capucins (dossier Mgr Persico). 
qu'il a écrit ou qu'il écrit actuellement quelque chose à la Propagan$\mathrm{de}^{25}$.

L'abbé Alexis Pelletier, qui fut mêlé à plusieurs disputes célèbres de ce temps, affirme : "Mgr Persico... a été en 1872 un envoyé secret de la Propagande au Canada, chargé d'étudier sur place la situation religieuse du pays et d'informer cette Congrégation ${ }^{26}$. "

Quelques voix américaines abondent dans le même sens; ainsi déclare M. Maurice Francis Egan : « He was sent to Canada on a mission of diplomacy ${ }^{27}$." Un autre Américain soutient : " In 1874, he was sent as Apostolic delegate to Canada ${ }^{28}$. "

Tous ces témoignages, contemporains ou postérieurs - et ils sont nombreux d'Asie, d'Europe, d'Amérique - sont unanimes à attribuer à Mgr Persico une mission d'informateur secret envoyé par le Vatican pour étudier les problèmes religieux de notre Province.

Mais ces affirmations, si autorisées soient-elles, sont loin de constituer des preuves; en feuilletant les archives, avec patience et minutie, j'ai eu l'heur de découvrir quelques textes de première importance dont je vous fais part.

Le 14 mai 1876, Mgr Persico fait cette confidence à l'abbé Benjamin Paquet : " Je suis décidé de quitter le Canada avant le mois d'octobre, par conséquent je dois résigner ma cure dans le mois de juillet... Il y aura un autre avantage dans cet état de liberté, et c'est que je pourrais rendre plus de services à la Sacrée Congrégation, observer, examiner, etc, et ainsi travailler à tête reposée et préparer un rapport secret et complet pour la Sacrée Congrégation ${ }^{29}$."

Je me suis adressé à la Propagande pour obtenir ce rapport, mais en vain, car les archives du Saint-Siège sont inaccessibles pour les cent ans postérieurs aux faits. On m'a fait alors cette réponse significative : "Ce rapport est trop compromettant pour les évêques Canadiens." Tout de même, je vous fais lecture d'un autre document d'une extrême importance qui authentifie bien la mission de Mgr Persico : " A Mgr Persico, Saint-Colomb-de-Sillery, 1 mai 1876. De divers côtés, et dernièrement selon Votre Révérence en a écrit à Mgr Roncetti, j'ai appris avec déplaisir les très graves questions qui ont été soulevées là-bas, au grand scandale des fidèles et avec un non moindre dommage pour l'Eglise, par l'ingérence de certains évêques et du clergé dans les élections politiques. Un tel état de choses ne peut pas ne pas réclamer mon

25 Lettre de l'abbé J.-B.-Z. Bolduc, Québec, à Mgr Benjamin Paquet, Rome, Arch. Univ. Laval, 112-BG.

26 Voix canadiennes, Vers l'abîme, éd. Arthur Savaète, tome III, p. 115 (voir note).

27 The Hierarchy of the Roman Catholic Church in the United States, ed. by Maurice Francis Egan, Philadelphia, George Barrie, Publisher, 1888, vol. I, p. $30-32$.

28 The Catholic Historical Review, Washington, D.C., 1916, vol. I, p. 377.

29 Arch. Univ. Laval, 113-BE. 
attention, et il exige qu'on y apporte quelque remède efficace. Pour le moment, parmi les moyens qui conviendraient peut-être au besoin, je croirais que l'un pourrait être d'envoyer au Canada un représentant du Saint-Siège, lequel résiderait à Québec et pourrait donner une direction aux évêques, tant pour les questions actuelles que pour celles qui pourraient survenir par après.

* A cette fin, je m'adresse sous le plus grand secret à Votre Révérence, l'invitant à rédiger une relation détaillée sur les inconvénients en général, et en particulier sur ceux que l'on déplore dans ces contrées lointaines, et à exprimer en même temps son sage avis sur l'opportunité et la convenance de l'expédient que l'on penserait adopter, ainsi que la manière selon laquelle Votre Révérence croirait opportun qu'on le devrait mettre en pratique, et sur les difficultés qu'il pourrait rencontrer.

"Dans l'attente d'une réponse, etc...30 *

Muni d'un tel mandat, Mgr Persico rédigea son rapport et le remit personnellement au Cardinal Secrétaire de la Propagande. En attendant le moment de consulter ce précieux document, nous pouvons connaître tout de même la pensée de Mgr Persico sur les événements religieux controversés de l'époque par les quelques allusions qu'il fit à ses intimes dans sa correspondance; ainsi confiait-il à l'abbé Benjamin Paquet : "Sur l'affaire Nicolet, j'ai des raisons spéciales de pouvoir donner mon opinion là-dessus. Comme il y a division entre les évêques au sujet de Nicolet, la Sacrée Congrégation devrait également avoir une opinion impartiale avant de donner une décision. Dans ma réponse à la lettre confidentielle, je parlerai aussi de l'ingérence dans les affaires politiques et au mal que cela fait... La Propagande a déjà assez de raisons pour nommer un délégué au Canada, s'il n'y avait autre chose que le vacarme de la politique, cela devrait suffire. Pour le moment, je ne vous dis autre chose ${ }^{31}$.

Mgr Persico, bien que retiré dans sa cure de Sillery, suit tous les événements de la scène canadienne avec beaucoup d'intérêt. L'abbé J.-B.-Z. Bolduc nous dit à ce propos : "Il connaît toutes nos affaires, aussi bien que nous; il a un jugement si droit, il aime tant le Canada, que je suis persuadé qu'il sera pour nous l'ange de la paix. » Prévoyant le départ prochain du prélat, il ajoute : "Pour nous, nous serions privés d'un ami sincère, dévoué, d'un conseiller sage et prudent ${ }^{32}$. »

Mgr Taschereau, qui soupçonne sans doute la mission du prélat romain, déclare : " Je profite du séjour de Mgr Persico ici pour le mettre au fait de bien des documents qui prouvent avec quel soin j'ai essayé de conduire la barque, tout en prenant l'avis des autres évêques,

30 Traduction de l'italien par le père Camille BÉRuBÉ, o.f.m.cap.

31 Lettre de Mgr Persico, Sillery, à Mgr Benjamin Paquet, Rome. Arch. Univ. Laval, 113-C.

32 Lettre de l'abbé J.-B.-Z. Bolduc, Québec, à l'abbé Benjamin Paquet, Rome, 21 juillet 1876. Arch. Univ. Laval, 114-T. 
et combien j'ai tenu à respecter les bornes posées par nos Conciles $^{33}$....

Ailleurs il poursuit : « J'écris aujourd'hui au cardinal (Cardinal Patrizzi) une longue lettre dont je donne une copie à Mgr Persico qui part demain. J'ai remis à ce brave Seigneur divers autres documents concernant les recommandations faites au clergé sur la politique, tant par les Evêques et par moi, que par le Saint-Siège ${ }^{34}$.

Mgr Persico est au courant non pas seulement par des écrits, mais

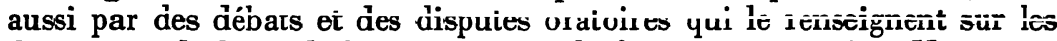
deux côtés de la médaille et, souvent, de façon très animée. Une scène fort cocasse nous le démontre : "Samedi dernier, raconte Mgr Taschereau, le Séminaire a donné un dîner d'adieu à Mgr Persico. Mgr Lynch et Mgr Cazeau sont tombés sur la politique dans un petit badinage, puis la mêlée est devenue générale, si bien que Mgr Persico, affligé et effrayé, s'est esquivé doucement... Je suis bien aise qu'il ait eu cette occasion de voir combien parmi nous une étincelle suffit pour mettre le feu à la poudre et causer des explosions terribles. Il pourra en donner des nouvelles aux Romains ${ }^{35}$."

Pour mieux remplir sa mission d'observateur secret, Mgr Persico se produit en public sans le moindre apparat vestimentaire. Voici le témoignage d'une de ses confirmées du Collège de Bellevue qui nous fournit en outre une description sommaire de lui : « Mgr Persico était de taille moyenne, aux épaules bien prises, figure ronde et colorée, teint clair, ne portant pas de barbe. En dehors du sanctuaire, il ne portait pas les insignes de sa dignité épiscopale; il faisait aux élèves l'effet d'un prêtre ordinaire ${ }^{36}$.

Cependant, Mgr Persico, malgré son désir d'effacement et de solitude, est l'objet de critique et de blâme de la part des conservateurs qui l'accusent de prendre le parti des libéraux. Ainsi, après son départ, s'exprime Mgr Laflèche qui demande : "Un Délégué Apostolique pour enquêter sur les affaires religieuses du Canada, et un délégué apostolique impartial et désintéressé et qui ne sera pas circonvenu par les libéraux comme l'avaient été auparavant Mgr Persico et Mgr Conroy ${ }^{37}$. "

Quoiqu'il en soit de cette affirmation, il demeure certain, comme nous l'apprennent les documents contemporains que j'ai apportés, que Mgr Persico fut chargé d'une mission secrète par le Saint-Siège. Cette mission, selon le témoignage même du prélat, consistait à a observer,

33 Lettre de Mgr Taschereau, Québec, à Mgr Benjamin Paquet, Rome, 6 octobre 1876. Arch. Univ. Laval, 115-AW.

34 Ibid., 13 octobre 1876, 115-BE.

35 Ibid.

36 Lettre de Mlle Odile Gilbert au père Justin. Arch. Prov. des Capucins, Montréal (dossier $\mathbf{M g r}$ Persico).

37 Le Patriote, Montréal, 6 décembre 1934, p. 3. Voir aussi : Voix cana. diennes, Vers l'abîme, op. cit., tome III, p. 105. 
examiner et préparer un rapport secret et complet pour la Sacrée Congrégation ${ }^{38}$.

Sans doute, il ne fut pas chargé d'une mission officielle avec le titre de Délégué Apostolique, car jusqu'alors la chose ne s'était jamais produite au Canada. Mais, en attendant le dépouillement des archives du Vatican, on peut affirmer que Rome profita de la venue et du séjour de Mgr Persico à Québec pour lui demander d'étudier la situation religieuse de notre pays et de lui en faire rapport. Il fut donc, de vive voix et par écrit, un informateur officieux de la Propagande sur les difficultés entre Montréal et Québec, au sujet de l'Université Laval et de sa succursale dans la Métropole. De plus, des indices non équivoques nous précisent qu'il l'informa également sur les difficultés politico-religieuses de 1870 à 1876 relatives au libéralisme et aux élections dans la Province de Québec, et sur l'opportunité d'ériger Nicolet en diocèse.

Mgr Persico a rempli sa mission avec fidélité, tact et impartialité pour le bien de l'Eglise et la paix de notre pays. Pendant trois ans, il abandonna les honneurs et les dignités ecclésiastiques pour se réfugier dans l'humble presbytère d'une paroisse canadienne. A Sillery, Mgr Persico laissa sa marque par son zèle et son dévouement auprès des petites gens, des malades, des pauvres et des enfants. Par ce seul fait, il aurait droit à notre admiration; mais ce prélat, en raison de sa dignité épiscopale, fut appelé à jouer un rôle plus grand, à la dimension même de notre immense pays. Son action apostolique et diplomatique, très appréciée à Rome, fut grandement utile à notre pay's qu'il a aimé comme le sien. * Après Naples, c'est Sillery ${ }^{89}$, , disait-il, et longtemps après son départ il répétait encore « que les trois années qu'il desservit cette paroisse furent les plus heureuses de sa vie ${ }^{40}$. La ville de Sillery, reconnaissante, perpétua sa mémoire en donnant son nom à une rue. Pour sa part, la Société canadienne d'histoire de l'Eglise catholique a voulu lui rendre hommage en mettant en lumière la mission secrète que cet évêque capucin a remplie parmi nous. Puisse le centenaire de son arrivée à Québec être l'occasion d'une plus grande célébration en son honneur.

\author{
Gabriel-Marie Dumas, o.f.m.cap., L. ès L., M.A., B.Bibl. \\ secrétaire et archiviste provincial \\ Provincialat des Capucins, Montréal, P.Q.
}

\title{
BIBLIOGRAPHIE A CONSULTER
}

- Il Cardinale Ignazio Persico, dei Cappuccini di Napoli et terra di Lavoro, appunti biografici, par F. Bonaventura DA SorRento, capucin, évêque de S. Severo (Italie).

- Ignatius cardinal Persico, par Donald Shearer, o.cap., New York, 1932, dans Franciscan Studies, $\mathrm{n}^{\circ} 10$ (1932), p. 53-137.

- Lexicon Capuccinum, promptuarium historico-bibliographicum Oridinis Fratrum Minorum Capuccinorum (1525-1950), Romæ, Bibliotheca Collegii Internationalis S. Laurentii Brundusini, 1951, 1862 col., voir col. 1334.

- Fr. Cuthbert, Persico, Ignatius Cardinal, in The Catholic Encyclopedia, 15th edition, New York, Robert Appleton Co., 1911, voir vol. XI, p. 725.

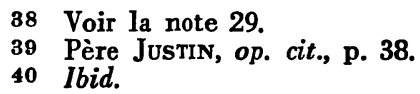

